\title{
A Research on High-Power LED Lighting Drive Based on Buck-Boost Converter
}

\author{
Renbo $\mathrm{Xu}^{1,2}$, Shaoyi $\mathrm{Li}^{1}$ and Zhilin Ding ${ }^{1}$ \\ School of artificial intelligence, Nanchang Institute of Science and Technology, Nanchang 330108, \\ china. \\ 2.Physics and Microelectronics Institute, Central South University, Changsha 410083 china
}

Keywords: Buck-Boost; High Efficiency and High Power; PID Correction; Optimized Lab

\begin{abstract}
This paper studies on a buck-boost high efficiency semiconductor lighting driver optimization design. Firstly, the structure principle of Buck-Boost drive circuit is discussed in detail, such as the working mode under the dynamic change of input and output voltage and current load to analyze the main circuit voltage mode control system. By using PID control technology, a new buck/boost voltage mode control system for PID correction network is created through theoretical setting analysis. After optimization and innovation, the system response speed, robustness, and adaptability to external environmental changes are greatly improved.
\end{abstract}

\section{Introduction}

Buck-Boost converter, also known as step-down and step-up converter, its basic idea is to connect a Boost converter with a Buck converter which can achieve buck and boost reverse polarity output and can overcome the problem that single Buck or Boost can only step down or step up voltage and avoid the single buck driver input current problem when the supply voltage is low. This kind of converter has a great deal of advantages, good performance, and is widely used in the drive control system. This article will study on a new type of high-power LED drive system based on the Buck-Boost converter. In the CCM mode, the state-space average method is used to construct the small-signal model of the main circuit. The closed-loop small-signal model of the entire system is established under the condition of voltage mode control, and the entire closed-loop system is corrected and optimized by using PID control technology and engineering setting method.

\section{Buck-Boost Semiconductor Lighting Drive Circuit Analysis}

The Buck-Boost type LED driver is a step-down step-up non-isolated driving converter, which can adjust the voltage by changing the duty cycle of the switching signal and also change the polarity of the output voltage, complete the reverse polarity voltage output and is widely used in various LED drivers. As shown in Figure 1 below, a Buck-Boost high-power LED driver circuit consists of an input voltage $u_{i n}$, a switch $Q$, an inductance $L$, a diode $D$, an output capacitor $C$, and a LED load.

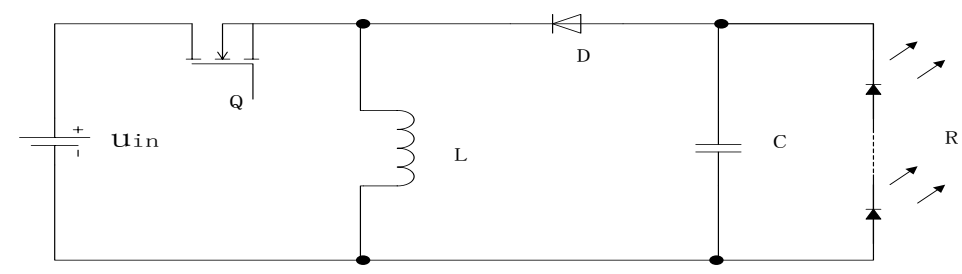

Figure 1 Buck-Boost High Power LED Driver

Because of the difference of the switch drive signal, the circuit can work in both continuous conduction mode and discontinuous conduction mode. The switch $Q$ is responsible for conducting, the input voltage is applied across the inductance $L$, and the current increases linearly. The inductance $L$ converts the energy into magnetic energy. At this time, the diode $D$ is reversed and the capacitor $C$ releases energy to the LED load. The circuit differential equation is shown in 
equation (1):

$$
\left\{\begin{array}{l}
u_{i n}=L \frac{d i_{L}}{d t} \\
\frac{u_{o}}{R}=-C \frac{d u_{o}}{d t}
\end{array}\right.
$$

The switch $Q$ is turned off and the diode $D$ is turned on. The inductance $L$ discharges through the diode $D$, capacitor $C$, and LED load. The capacitor $C$ is charged and the current is continuously reduced, but it will not drop to zero until the switch $Q$ turns on again until the arrival of the next switching cycle. Its circuit differential equations are shown in equation (2):

$$
\left\{\begin{array}{l}
-u_{o}=L \frac{d i_{L}}{d t} \\
i_{L}=C \frac{d u_{o}}{d t}+\frac{u_{o}}{R}
\end{array}\right.
$$

When the inductance $L$ is releasing energy through the diode $D$, capacitor $C$, and LED load, $L$ decreases linearly. When the inductance $L$ gradually decreases and is greater than the output current, the energy on the inductance $L$ is transmitted to the load and the capacitor $C$, the load LED emits light, the capacitor $C$ charges and electrical energy is stored. When the inductance $L$ is equal to the output current and continues to decrease and is not zero, the inductance $L$ continues to release energy to the load, and the capacitor $C$ changes to the discharged state, and together with the inductor current, supplies power to the load LED and transfers the energy to the load. Before the current drops to zero, the next cycle signal arrives and the inductor current is charged again to store energy. This mode of operation is called the CCM mode. This mode can be subdivided into a single inductance supply mode and a common inductance and capacitor supply mode. After the inductor current drops to zero, the periodic signal has not yet arrived, and the inductance cannot charge while maintaining zero current. The entire driver circuit will enter the capacitor-to-load LED power supply mode, which is called DCM mode.

After knowing the transfer function of the rest of the loop, an error amplifier $G_{c}$ design can be performed. As the "control-output" function provides a zero point and two resonant poles, from the system model block diagram, we can know that the voltage mode control Buck-Boost LED drive converter has the following drawbacks: (1) When there is no prediction for input voltage disturbance based on voltage feedback mechanism, the input voltage has slow response, which requires a high loop gain and asks for higher requirements for the system. (2) Since the two resonant poles are not compensated, the dynamic response performance is poor. This paper uses PID correction network to compensate, to eliminate the system steady-state error, increase the cut-off frequency, make the open-loop gain at the cut-off frequency cross the $0 \mathrm{~dB}$ line at $-20 \mathrm{~dB} / \mathrm{decade}$, increase the phase margin, make the phase margin slightly less than $90^{\circ}$.

\section{System Optimization and Correction Design of Buck-Boost Type Driver}

The system optimization correction is based on automatic control technology, and most of the automatic control technology utilizes a feedback loop for compensation. In the feedback theory, the measured variable or output value is compared with the expected value or the reference value, and the generated error signal is fed back to the system to adjust and correct the response of the system so that the response reaches the desired value. Most of the control loops in the process control use the PID structure, and many advanced automatic controls are also based on the PID structure. In this paper, PID control technology is used to compensate and correct the Buck-Boost type LED driving converter system. The PID controller is often connected in series in the system channel to play the role of series correction, and it can differentiate between two types of PID 
control systems with series lead and series hysteresis based on its phase-frequency characteristics leading or lagging. Figure 2 shows the structure of the control system consisting of a PID controller.

Among them, $G_{c}(s)$ is the transfer function of the controller, $G_{0}(s)$ is the transfer function of the inherent part of the system.

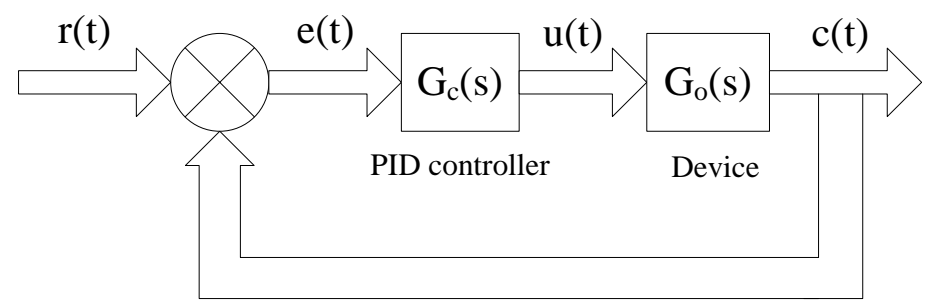

Figure 2 The structure of the control system consisting of a PID controller

PID control is a kind of control law formed after mathematic operation of deviation signal $e(t)$. That is, the controller output is:

$$
u(t)=K_{p}\left[e(t)+\frac{1}{T_{i}} \int_{0}^{t} e(t) d t+T_{d} \frac{d}{d t} e(t)\right]
$$

In the formula, $K_{p} e(t)$ is a proportional control item and $K_{p}$ is called a proportional coefficient;

$\frac{1}{T_{i}} \int_{0}^{t} e(t) d t$ is integral control term, $T_{i}$ is called integral time constant;

$T_{d} \frac{d}{d t} e(t)$ is a derivative control term $T_{d}$ is called differential time constant.

\section{System Control Theory Setting Analysis}

In order to facilitate the PID parameter setting, we set $K_{f b}=1, G_{m d}=1$, and the open-loop transfer function of the Buck-Boost type LED drive converter is:

$$
G_{O}(s)=\frac{\left[L D s-R(1-D)^{2}\right] U_{\text {in }}}{\left[R L C(1-D)^{2} s^{2}+L(1-D)^{2} s+R(1-D)^{4}\right]}
$$

According to the previous parameters, the PID theory analysis is performed. Here, the MATLAB programming study is performed using proportional differential control (PD) and proportional integral control (PI). The system characteristics are shown in Figure 3. 


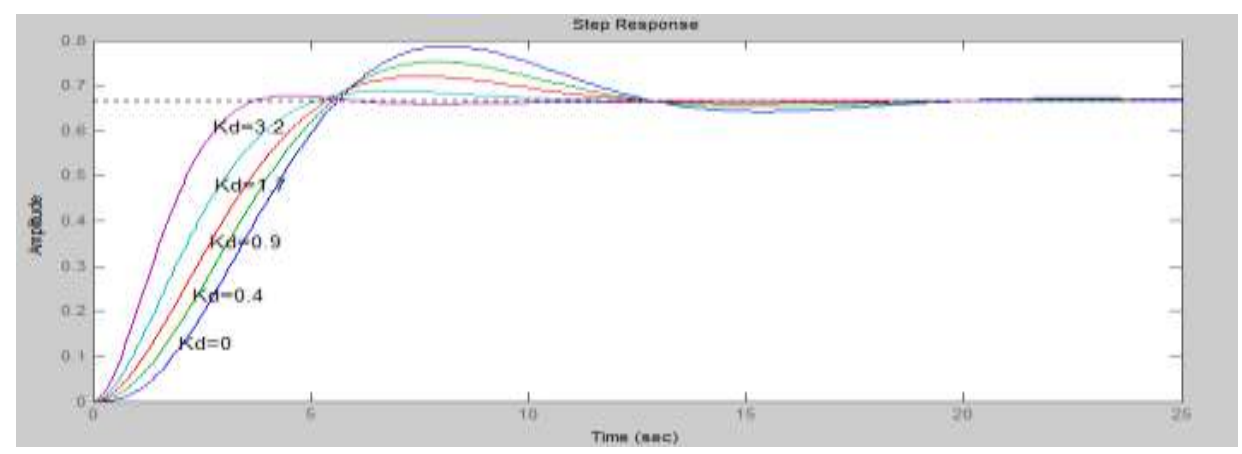

Figure 3 PD step response of Buck-Boost LED driver

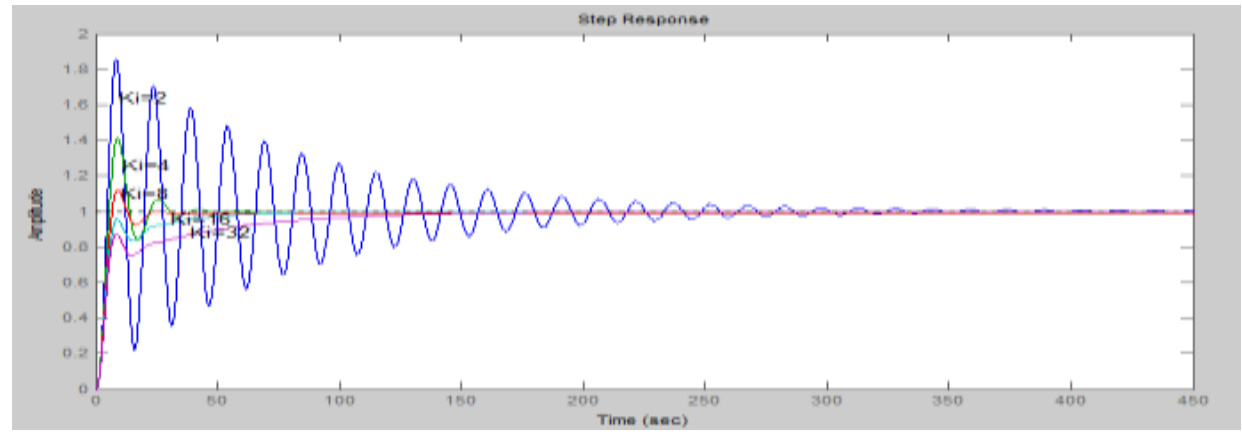

Figure 4 PI step response of Buck-Boost LED driver

From Fig. 3 and Fig. 4, it can be seen that as the differential coefficient increases, the differential action increases continuously, the system overshoot is reduced, the system stability is improved, the rise time is reduced, and the system rapidity is improved; With the constant decrease of the coefficient, the integral action is continuously enhanced and the stability of the closed-loop system is deteriorated, but the system error is reduced. To combine the advantages of both, using PID control improves system stability and rapidity while also providing better dynamic performance.

\section{Conclusion}

This paper studies on a buck-boost high efficiency semiconductor lighting driver optimization design. The structure principle of Buck-Boost drive circuit is discussed in detail, such as the working mode under the dynamic change of input and output voltage and current load. It also analyzes the main circuit voltage mode control system. The PID correction network is adopted to comprehensively improve the control performance of the voltage mode control of the Buck-Boost type LED drive converter. The introduced differential sector can predict the change trend of the system output voltage to compensate for the time delay caused by the low-pass filter, which can improve the system's response to the input and suppress the ability to overshoot, so that the system's dynamic characteristics and load characteristics have been greatly improved.

\section{Acknowledgement}

Project Funding: Jiangxi Educational Science Planning Topics (No.17YB252); Nanchang DME Photoelectric Engineering Key Laboratory (No.NCZDSY-004); Nanchang Institute of Science \& Technology Research Fund (No. NGRCZX-17-01)

\section{References}

[1] Jinbang Xu, Jin Zhao, Ling Luo. A new control strategy of unity power factor for three-phase PWM rectifier system. Industrial Electronics Society, 30th Annual Conference of IEEE, 2004, 25(7):709-714.

[2] Wenkai Wu, Weihong Qiu. Efficient high power factor single-stage AC/DC converter. IEEE, 2012, 28(4):1143-1148. 
[3] Xiaoqun Wu, Chi K. Tse. Fast-Scale Instability of Single-Stage Power-Factor-Correction Power Supplies. IEEE, 2008, 37(5):477-480.

[4] J. P. Noon. Designing High-Power Factor Off-Line Power Supplies. Proceedings of Unitrode power Supply Design Seminar Manual SEM1500, Texas Instruments, 2007, 43(2):2-6.

[5] H. van der Broeck, G. Sauerlander, M. Wendt. Power Driver Topologies and Control Schemes for LEDs. IEEE Applied Power Electronics Conference, Anaheim, 2007, 31(5):319-325

[6]B. Wang, X. Ruan, K. Yao. A Method of Reducing the Peak-to-Average Ratio of LED Current for Electrolytic Capacitor-Less AC-DC Drivers. IEEE Transactions on Power Electronics, 2010, 25(3): 592-601

[7] E. Najafi, A. Vahedi, A. Mahanfar etal. A new controlling method based on peak current mode (PCM) for PFC. IEEE 2nd International Power and Energy Conference, 2008, 51(3):1103-1107. 